\title{
Permeability, Solubility, and Interaction of Hydrogen in Polymers- An Assessment of Materials for Hydrogen Transport
}

\author{
M.C. Kane
}

Savannah River National Laboratory

Washington Savannah River Company

Publication Date:

Washington Savannah River Company

Savannah River Site

This document was prepared in connection with work done under Contract No. DE-AC09-96SR18500 with the U. S. Department of Energy 


\section{DISCLAIMER}

This report was prepared as an account of work sponsored by an agency of the United States Government. Neither the United States Government nor any agency thereof, nor any of their employees, makes any warranty, express or implied, or assumes any legal liability or responsibility for the accuracy, completeness, or usefulness of any information, apparatus, product, or process disclosed, or represents that its use would not infringe privately owned rights. Reference herein to any specific commercial product, process, or service by trade name, trademark, manufacturer, or otherwise does not necessarily constitute or imply its endorsement, recommendation, or favoring by the United States Government or any agency thereof. The views and opinions of authors expressed herein do not necessarily state or reflect those of the United States Government or any agency thereof. 
DOCUMENT: WSRC-STI-2008-00009, Rev. 0

TITLE: $\quad$ Permeability, Solubility, and Interaction of Hydrogen in Polymers- An Assessment of Materials for Hydrogen Transport

\section{APPROVALS}

M.C. Kane, Author

Date:

SRNL-MATERIALS SCIENCE \& TECHNOLOGY

Materials Applications \& Process Technology Group

E.A. Clark, Technical Review

Date:

SRNL-MATERIALS SCIENCE \& TECHNOLOGY

Materials Composition and Welding Technology

G.B. Rawls, Task Lead

SRNL-MATERIALS SCIENCE \& TECHNOLOGY

Materials Applications \& Process Technology Group

R.L. Sindelar, Manager

SRNL-MATERIALS SCIENCE \& TECHNOLOGY

Materials Applications \& Process Technology Group

N.C. Iyer, Director

Date:

SRNL-MATERIALS SCIENCE \& TECHNOLOGY

Date:

Date: 


\section{Table of Contents}

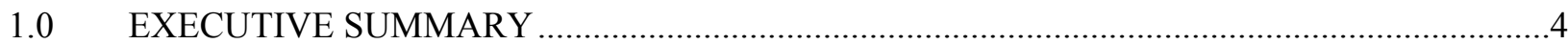

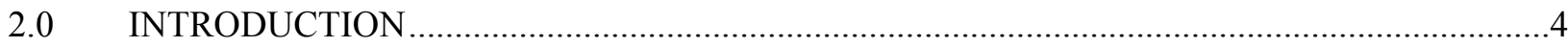

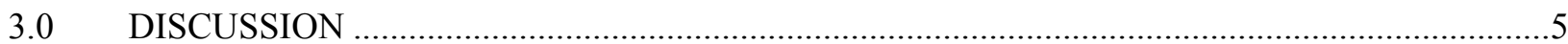

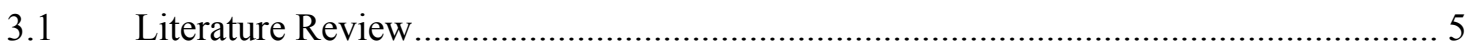

3.1.1 Permeability Trends in Molecular Gas Diffusion in Polymers.................................. 6

3.1.2 Permeability, Solubility, and Diffusion Measured ............................................. 7

3.1.3 Potential Degradation of Polymers in Hydrogen ................................................... 9

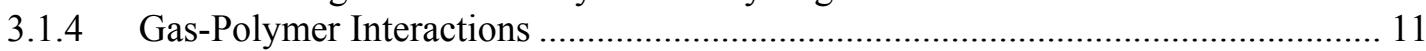

3.1.5 Predicting Polymer Permeability Behavior ............................................................... 12

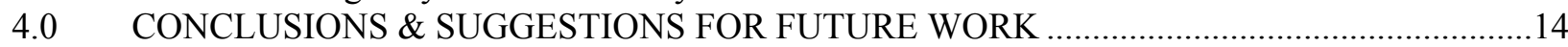

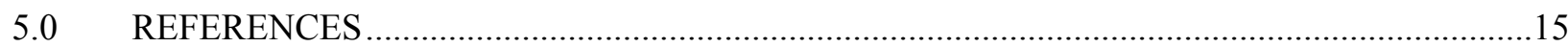

6.0 APPENDIX- BASIC MODEL FOR HYDROGEN TRANSPORT IN POLYMERS ..................17 


\subsection{EXECUTIVE SUMMARY}

Fiber-reinforced polymer (FRP) piping has been identified as a leading candidate for use in a transport system for the Hydrogen Economy. Understanding the permeation and leakage of hydrogen through the candidate materials is vital to effective materials system selection or design and development of safe and efficient materials for this application. A survey of the literature showed that little data on hydrogen permeation are available and no mechanistically-based models to quantitatively predict permeation behavior have been developed. However, several qualitative trends in gaseous permeation have been identified and simple calculations have been performed to identify leakage rates for polymers of varying crystallinity. Additionally, no plausible mechanism was found for the degradation of polymeric materials in the presence of pure hydrogen. The absence of anticipated degradation is due to lack of interactions between hydrogen and FRP and very low solubility coefficients of hydrogen in polymeric materials. Recommendations are made to address research and testing needs to support successful materials development and use of FRP materials for hydrogen transport and distribution.

\subsection{INTRODUCTION}

Fiber-reinforced polymer (plastic) piping has been identified as a leading candidate for the material system to transport and distribute hydrogen gas for the broad use of the Hydrogen Economy ${ }^{1}$. Understanding the permeation, or flux, of hydrogen through polymeric materials is vital to the selection or development of appropriate materials for this application. In order to design an effective infrastructure for future hydrogen transport, a pipeline material must be developed or a commercial material identified that has minimal permeability to hydrogen and with sufficient mechanical properties to maintain the integrity of the pipeline during its lifetime. The cost must also be economically viable. Based on the current understanding of permeation through polymeric materials, commercially available polymers have been suggested for use and their performance in the presence of hydrogen can be predicted to a reasonable extent.

This report assesses the literature data/knowledge on the permeability behavior of potential fiber reinforced piping (FRP) liner materials in the presence of hydrogen and makes suggestions for future research and testing. The commercial pipeline materials of most interest are Fiberspar ${ }^{\circledR}$ and Poly-Flow. The liner in Fiberspar ${ }^{\circledR}$ is a commercial high-density polyethylene (HDPE) and that of Poly-Flow is polyphenylene sulfide (PPS). Although a limited number of studies on hydrogen permeability have been completed on polyethylene, polyphenylene sulfide (PPS) has not received any research attention with respect to gaseous permeation.

The piping liner material is considered to be the limiting factor for permeability of hydrogen. The outer pipe layer, which will most likely be a glass or aramid-fiber reinforced thermoset (e.g., epoxy), is very permeable to hydrogen gas due to the 
formation of microcracks during the spooling process and does not provide any barrier characteristics ${ }^{2}$.

\subsection{DISCUSSION}

\subsection{Literature Review}

Gaseous permeation through polymers has typically been studied for materials designed for use as either barrier materials (low permeability) or as membrane materials (high permeability/permselectivity). The majority of studies have focused on glassy (amorphous) polymers for membrane applications. Barrier materials typically have more flexible carbon-carbon backbones with highly polar pendant groups, while membrane materials generally have an aromatic, rigid backbone structure ${ }^{3}$.

There are a very limited number of studies on the permeation of hydrogen in polyethylene (PE), despite its proposed use as a barrier (liner) material for the hydrogen economy infrastructure. There are even fewer studies of the permeation of hydrogen of a PE pipe material used in gaseous transport service ${ }^{4}$. Semicrystalline polymers, such as polyethylene, have both amorphous and crystalline regions, making the prediction of permeation properties complicated due to the presence of two distinct phases. The permeability of polymers like PE to hydrogen will depend on the relative amounts of each phase as well as the size, shape, and distribution of the secondary phase. It has been shown that diffusion only takes place in the amorphous regions, which act like an amorphous liquid (above $\mathrm{T}_{\mathrm{g}}$ ), whereas the crystalline regions act as a barrier, restricting permeation completely ${ }^{5}$. With respect to permeability, it is clear that a highly crystalline polymer would be desirable for barrier applications, but other materials characteristics affect the performance characteristics and must be considered in the materials selection process.

There are several factors that affect the permeability of polymers to hydrogen, including crystallinity, chain orientation, fillers, and side chain complexity. All of these properties affect the free volume available for molecular diffusion of permeant species. Free volume is defined as the unoccoupied region included in the polymer volume that is accessible to polymer chain segmental motions. The presence of free volume enables molecular gas diffusion in that the diffusing species moves from one open "site" to another by chain movement into the free volume space. The available free volume can change drastically with minimal changes in polymer properties. Conversely, a polymer can be specifically engineered to maximize or minimize the free volume to tailor the permeability properties according to the application. A detailed list of general factors affecting gas permeability in polymers is given in the next section. 


\subsubsection{Permeability Trends in Molecular Gas Diffusion in Polymers}

Many polymer properties or characteristics have an impact on gaseous permeability in a polymer ${ }^{6}$. The general trends, according to the Polymer Handbook, are ${ }^{7}$ :

Polymer Type- In general, permeability decreases from elastomers to amorphous polymers to semi-crystalline polymers due to crystallinity and changes in the free volume.

Complex and bulky side chain species can hinder chain movement and decrease permeability compared to a completely linear polymer, such as polyethylene.

Density can be defined as a measure of the free volume between the molecules of the polymer structure. Thus, the higher the density, the lower is the permeability.

Crystallinity of a semicrystalline polymer (e.g., polyethylene) reduces the permeability significantly compared to the value of the corresponding amorphous polymer. The crystallinity and the density of a polymer are strongly related; the higher the crystallinity the higher the density of a given polymer. As the degree of crystallinity increases, the permeability decreases.

Molecular mass of a polymer has been found to have little effect on the permeability of polymers, except at a very low range of molecular masses.

Orientation or alignment of polymer molecules reduces the permeability. For example, an extruded polymer that exhibits chain alignment in the direction of extrusion may have a lower permeability than the same polymer that was solution cast.

Crosslinking decreases the permeability, especially for higher degrees of crosslinking (above the entanglement MW) and for permeants that are larger than the distance between crosslinks.

Glass transition temperature, $T_{g}$ - Permeation rates above $\mathrm{T}_{\mathrm{g}}$ are much higher than below $\mathrm{T}_{\mathrm{g}}$ due to free volume and chain movement.

Plasticizers increase the permeability. Small plasticizer molecules penetrate between the polymer molecules (chains) and reduce the density, thus decrease the $\mathrm{T}_{\mathrm{g}}$ of the polymer and increase permeability.

Humidity or water vapor in a gaseous stream increases the permeability of some hydrophilic polymers. A typical commercial grade of high density polyethylene is hydrophobic and naturally water-repellent. However, a hydophilic polymer may take up water into the bulk polymer, causing swelling, and a greater free volume available for permeation. 
Solution cast films have variable permeabilities depending upon the kind of solvent used and the drying technique. Poor solvents tend to yield films of higher permeability due to contracting polymer chains and greater free volume.

Fillers, usually inorganic fillers such as silica or clay, provide a physical barrier to diffusion and decrease the permeability. However, the effect is complicated by the type, shape, and amount of filler and its interaction with the polymer.

Thickness of film does not, in principle, affect the permeability, diffusion, or solubility coefficient. In practice, different values may be obtained from films of variable thickness, which may be due to differences in drawing, orientation, and crystallinity. However, the permeation flux is directly dependent on the film thickness (see Appendix).

Even though no specific structure-property relationships have been quantified, the general trends of gaseous permeation presented here are based on numerous studies. There is an obvious separation of permeation behavior between glassy polymers and semi-crystalline (rubbery) polymers. Gases can be divided into three groups: non-polar diatomic gases, polar gases, and fluorinated gases, each having distinct permeation behavior based on polarity and molecular size. Diatomic hydrogen gas, having one $\mathrm{H}-\mathrm{H}$ bond, does not have an induced dipole (uneven charge distribution across the bond) and is considered non-polar.

\subsubsection{Permeability, Solubility, and Diffusion Measured}

Permeability measurements have been made for years on polymeric materials by various techniques. The technique most often used currently is the time-lag method, where the permeating gas is introduced to one side of the film, often by an inert carrier gas, and a vacuum is maintained on the opposite side. A gas sensing device is used to determine the amount of gas (in this case, hydrogen) that permeates through the film as a function of time. The diffusion coefficient can be directly related to the thickness of the film and the time it takes to reach steadystate gas flux, or time-lag (see Appendix). Apparent values for the diffusion and solubility coefficients can be readily obtained by this method, especially if there is little interaction between the polymer and the permeating gas ${ }^{4}$.

Solubility of a gas in a polymer involves an intimate interaction between the polymer and gas molecules. The solubility is determined by the balance of intermolecular forces between the polymer and gas and the entropy change that accompanies the solvation. Diffusion is a purely physical process of gas molecule movement through the polymer chains via free volume. This would imply that solubility is based on chemical interactions, while diffusion is purely physical movement of molecules through the material with little or no interaction between the polymer and the permeating species. 
In order to demonstrate that the main factor in determining the hydrogen permeability properties at a given temperature are predominantly controlled by changes in diffusion (physical movement) and not solubility (molecular interaction), the solubility coefficients of many polymers are presented. The measured solubility of hydrogen in a semi-crystalline (rubbery) polymer, such as HDPE, is comparatively low and will be similar for polymers of similar extent of crystallinity, as indicated in Figure 1. Permeation measurements conducted at Oak Ridge National Laboratory (ORNL) on a variety of polymers, including HDPE, polyether ketoneketone (PEKK), polyethylene terepthalate (PET), and polyvinildine fluoride (PVDF, Kynar ${ }^{\circledR}$ ) showed this to be true. All of these polymers are semi-crystalline thermoplastic resins that can be extruded into piping materials, yet have varied chemical structures. The calculated solubility coefficient varied little over the polymers tested (at a given temperature).

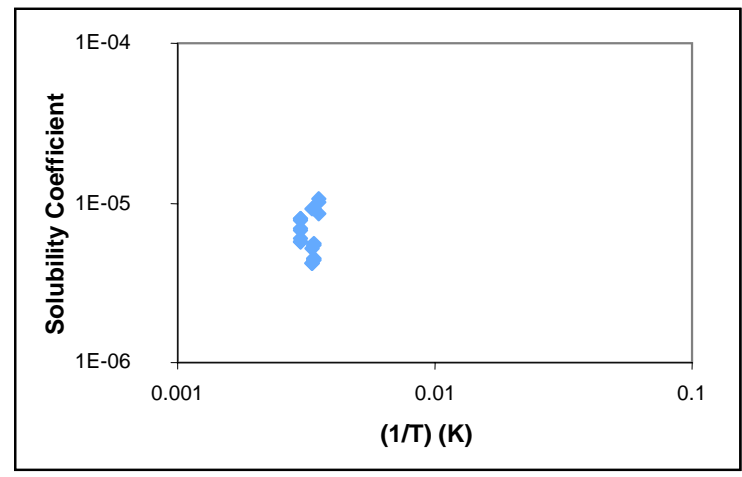

Figure 1. Adapted from ORNL data (B. Smith, B. Frame, et al, Pipeline Working Group Meeting, Aiken, SC, September 25-26, 2007) from several polymers tested for solubility coefficient, including HDPE, PEKK, PET and Kynar®, all with similar solubility values.

This similarity in solubility finding was also verified by Tanaka and coworkers ${ }^{8}$, who found that the variation in the permeability coefficient, $P$, was mainly due to the change in the diffusion coefficient, $D$, for all polyimide polymers investigated and not the solubility. The small variability (less than one order of magnitude) in the solubility constant is shown in Table 1. These findings point out that despite variability in the chemistry of the polymers tested, there is little change in the solubility coefficient; thus, there is little interaction between the polymer and permeating gas and diffusion is the limiting factor in permeability.

The diffusion coefficient varies from one polymer type to another much more than the solubility parameter, with free volume being the most influential parameter $^{3-6}$. Table 1 illustrates that the standard deviation for the diffusion coefficient is more than ten times larger than that of the solubility coefficient for the polyimides tested with hydrogen at a given temperature $\left(35^{\circ} \mathrm{C}\right)$, thus further indicating that permeation is driven by diffusion and not gas-polymer interactions that would influence changes in the solubility. 


\begin{tabular}{cccc}
\hline Polymer number & $P\left(10^{-10}\right)$ & $D\left(10^{-7}\right)$ & $S\left(10^{-3}\right)$ \\
\hline 1 & 5.92 & 2.7 & 2.2 \\
2 & 10.6 & 7.9 & 1.4 \\
3 & 34.3 & 22 & 1.5 \\
4 & 50.4 & 22 & 2.3 \\
5 & 3.68 & -- & -- \\
6 & 17.3 & 5.8 & 3.0 \\
7 & 30.6 & 10 & 3.0 \\
8 & 59.1 & 40 & 1.5 \\
9 & 4.79 & 3.6 & 1.3 \\
10 & 16.1 & 11 & 1.5 \\
11 & 24.6 & 8.4 & 2.9 \\
12 & 30.8 & 6.7 & 4.6 \\
13 & 23.7 & 15 & 1.6 \\
14 & 38.2 & 22 & 1.7 \\
15 & 40.7 & 24 & 1.7 \\
16 & 47.4 & 46 & 1.0 \\
17 & 55.4 & 12 & 4.6 \\
18 & 108 & 29 & 3.7 \\
\hline Std. Dev. & 25.4 & 12.6 & 1.13 \\
\hline
\end{tabular}

Table 1. Permeation, diffusion, and solubility coefficients of several fluorinated polyimides ( $P$ is in $\left.\mathrm{cm}^{3}(\mathrm{STP}) \mathrm{cm}^{-1} \mathrm{~s}^{-1} \mathrm{cmHg}^{-1}\right)$. Adapted from Tanaka, et. al (1992).

This conclusion was further verified by Klopffer, et $\mathrm{al}^{4}$ that over the various gases and gas mixtures tested for permeation through semi-crystalline polymers like polyethylene, there were no gas-gas or gas-polymer interactions detected, regardless of gaseous concentration. Wang, et. $\mathrm{al}^{9}$ also concluded that $\mathrm{H}_{2}, \mathrm{~N}_{2}$ and $\mathrm{O}_{2}$ are non-polar gases, thus, there are no special interactions between the gas and polymer backbone.

\subsubsection{Potential Degradation of Polymers in Hydrogen}

The stability of a polymeric material during service is essential for reliability. It is for this reason that all possible polymer degradation processes during hydrogen pipeline operating conditions must be known and mitigated. Polymer degradation due to elevated heat and stresses is well documented; however, degradation due to gaseous permeation, specifically hydrogen, is not widely researched and is of major interest for the selection of materials for fiber-reinforced piping for hydrogen service.

Polymer degradation can occur with exposure to normal environmental conditions, such as sunlight and oxygen. Samples exposed to UV light tend to continue to oxidize even when stored in darkness. In general, degradation usually occurs due to 1) irradiation and subsequent formation of free radicals within the polymer, 2) chemical attack of certain functional groups in the polymer chain possibly by changing $\mathrm{pH}$ conditions or humidity, or 3) thermal breakdown of polymers that are above $\mathrm{T}_{\mathrm{m}}$ or do not have a melting temperature due to physical crosslinking. Often, a combination of these three factors leads to polymer breakdown $^{10-13}$. 
Polyethylene, for example, can be degraded by heating (in an inert atmosphere) to approximately $450^{\circ} \mathrm{C}$. The breakdown is a result of random chain scission of the polymer backbone ${ }^{10}$. Random chain scission has been mentioned as the most important degradation mechanism, especially in polymers with aliphatic $\mathrm{C}-\mathrm{H}$ bonds $^{12}$. Chain scission also occurs due to irradiation. Polymers may experience degradation in the presence of water due to hydrolysis of the polymer molecules ${ }^{11}$. When chain scission occurs, by whatever catalyst, a ductile to brittle behavior change may be induced. This change is often termed as embrittlement and can drastically alter the properties of a polymer material. Embrittlement is due to many factors, including changes in crystallinity and molecular weight. However, degradation must occur before embrittlement takes place.

Crystallinity may change during the course of degradation. In the initial stages of photodegradation, chain scission often prevails, which reduces molecular weight. Shorter chains are more mobile and are thus able to crystallize more readily. Therefore, embrittlement of the polymer is driven by two associated processes: reduction of molecular weight and increased crystallinity. Additionally, degradation processes take place only in the amorphous regions of the polymer. Gaseous diffusion into the crystalline phases is restricted (if not prohibited completely), thus decreasing the potential for oxidation, and/or reaction with another permeating species, if the polymer has a high crystalline content.

The reaction pathway for radiation degradation of polyethylene (in the presence of $\mathrm{O}_{2}$ ) and the formation of free radicals is as follows ${ }^{13}$ : First, polyethylene forms weakly-absorbing complexes with ground-state molecular oxygen, which, on UV exposure, generate hydroperoxides. Next, transition metal ions are known to catalyze hydroperoxide decomposition. Both high-density PE and low-density PE contain unsaturated hydrocarbon bonds. The presence of these unsaturations (vinylidene groups) leads to the formation of allylic hydroperoxides during the thermooxidative processes, and this becomes the major mechanism of initiation. The resultant structure can be further converted by heat, UV, or other radicals to free radicals and/or to structures containing UV-absorbing groups (e.g., carbonyl). Cross-linking can also occur, but the chain scission mechanism most often dominates $^{13}$.

With respect to the investigation of degradation of a polyethylene liner in a pipeline for hydrogen service, no mechanisms for degradation due to hydrogen alone has been reported. Little or no interaction between hydrogen gas (or any non-polar gas) and polyethylene should be expected. Additionally, hydrogen alone provides no mechanism for radical formation, as mentioned previously for chain scission. However, if the permeating gas stream contained contaminants in addition to hydrogen gas, then the mechanism for degradation would depend solely on the contaminant concentration and nature of the contaminant. In some cases, contaminant gases like sulfur dioxide actually decrease the amount of hydrogen to permeate the polymer by essentially "plugging up" all of the free volume available for diffusion. 
Currently, specifications for the purity level of hydrogen gas transported via FRP pipeline have not been determined. However, it can be concluded that pure hydrogen gas will not promote polymer degradation, as mentioned previously. If, however, hydrogen gas is mixed with natural gas as a carrier (hythane), then the effects of natural gas on the stability of the pipeline liner material will become important. These findings have already been published elsewhere, although the main focus of these studies has been on the mechanical properties and not the degradation mechanisms ${ }^{2}$. Additionally, known gaseous contaminants, such as $\mathrm{CO}_{2}, \mathrm{H}_{2} \mathrm{~S}$, water vapor, chloride gas, and oxygen (among others) will also require further study into potential problems raised by the presence of these gases in the hydrogen stream.

\subsubsection{Gas-Polymer Interactions}

There is no mechanism for degradation of polyethylene in the presence of hydrogen unless some other reaction catalyst, such as heat, humidity, or radiation source is present. That is, any interaction between the hydrogen molecule and polyethylene chains would be very small, if at all. The concept of quantifying the degree of interaction between a polymer and another molecule (mainly a solvent or plasticizer) was first introduced by Flory and Huggins (simultaneously) in $1950^{6}$. The interaction parameter, $\chi$, was proposed as a single parameter to quantify the interactions between components in a mixture, which is related to the change in energy when the polymer/polymer and molecule/molecule (molecule $=$ solvent, plasticizer, permeant gas, etc) contacts are replaced by polymer/molecule contacts. This change in interaction energy can be expressed in the form of cohesive energy density and is related to solubility parameters ${ }^{14}$.

The $\chi$ parameter is usually expressed in terms of solubility of a polymer in a given solvent, but this parameter has been used recently to predict the solubility of a gas in the same way ${ }^{15-16}$. In the work of Kamiya ${ }^{15-16}$, hydrogen gas was termed a "sparingly soluble" gas in both polyethylene and poly(dimethylsiloxane) polymers by way of sorption isotherm measurements. As mentioned previously, if solubility is small, then the gas-polymer interactions can be described by Henry's Law. If not, then the Flory-Huggins theory of dissolution applies for rubbery polymers, while glassy polymers are described by the dual mode dissolution theory. In the case of hydrogen, Henry's Law was found to apply and a linear isotherm was observed ${ }^{4}$.

The $\chi$ parameter was estimated at around 3.1-3.5 for various grades of polyethylene, for the polyethylene/hydrogen interactions ${ }^{15-16}$. A value of 0.5 or below for this parameter indicates "good" solubility of the molecule in the polymer. An exact value of 0.5 indicates that the Flory theta $(\theta)$ condition was met. For this polymer-molecule pair, this means that the gas molecules are allowed to "flow" into and out of the polymer freely and with equal statistical probability without any thermodynamic restriction. A value of 0.5 and above indicates a poorly soluble molecule. The results of the work by the Kamiya group 
indicate that hydrogen is quantitatively very poorly soluble in polyethylene and other similar rubbery polymers.

A more rigorous investigation of the literature for generalizations on hydrogen interactions with polymers indicates that hydrogen interacts with rubbery polymers, in a similar manner as its interactions with a simple fluid, such as water. The solubility of hydrogen in water is well documented and known to be very low (Figure 2).

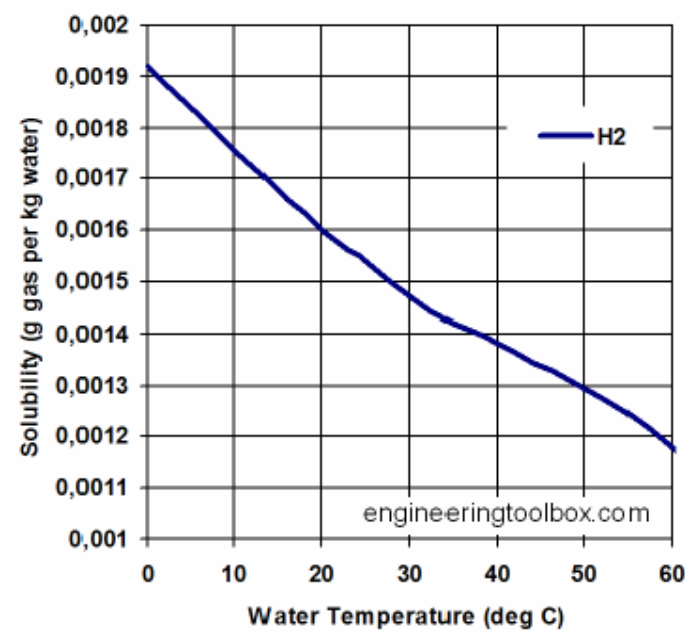

Figure 2. Solubility of hydrogen gas in water.

There are, however, a few instances when hydrogen can be thermodynamically "forced" to interact with a polymer. If hydrogenation takes place at or above certain critical conditions (temperature, pressure, etc), then the kinetic limitations to hydrogen solubility can be overcome. A "good" solvent that is hydrogenated can also be used to insert $\mathrm{H}_{2}$ molecules between polymer chains when the solvent is removed.

\subsubsection{Predicting Polymer Permeability Behavior}

When considering a single type of polymer, such as polyethylene, many commercial grades exist under the same chemical name. There is linear low density polyethylene (LLDPE), ultra high molecule weight polyethylene (UHMWPE), branched polyethylene, high density polyethylene (HDPE), etc. Each type has variation in the amount of crystallinity, cross-linking, density, branching, etc. If polyethylene was chosen as a suitable economical option as a liner material for fiber reinforced piping for hydrogen transport, there are many factors to consider. To avoid the rigor of testing each grade of polyethylene for permeability, the generic trends in permeability discussed above can be applied. Simple calculations that consider the volume fraction of amorphous regions within the polymer will reveal drastic changes in gaseous flux through the material. If, however, a more rigorous approach is desired, the interaction energy between the polymer and penetrant gas can be directly calculated according to the 
solution-dissolution model for rubbery polymers or the dual-mode dissolution model for glassy polymers.

To demonstrate the effect of crystallinity on permeation behavior of molecular hydrogen through a sample of polyethylene liner piping material, two sample calculations are presented for a highly amorphous polyethylene and highly crystalline polyethylene. The flux, or leakage rate is then calculated to determine the amount of hydrogen lost at typical service conditions.

From reference 15 , the solubility constant, $S$, for LDPE (42\% crystallinity by volume) is known and can be used to calculate $S^{*}$ for a completely amorphous polymer according to 5

$$
S=(1-\alpha) S^{*}
$$

where $\alpha$ is the volume fraction crystallinity of PE. The calculation for $S^{*}$ is as follows:

$$
\begin{aligned}
& 1.283 \times 10^{-7}\left(\mathrm{~Pa}^{-1}\right)=(1-.42) S^{*} \\
& S^{*}=2.212 \times 10^{-7}
\end{aligned}
$$

From this result, the solubility constant can be calculated for any polymer with crystallinity $\alpha$. For a hypothetical very low density polymer $(\alpha=30 \mathrm{vol} \%)$ and a very high density polymer $(\alpha=90 \mathrm{vol} \%)$, the calculated solubilities are $S=$ $1.55 \times 10^{-7}$ and $2.21 \times 10^{-8}\left(\mathrm{~Pa}^{-1}\right)$, respectively.

Using these calculations for solubility and the diffusion coefficient measured by Humpenöder ${ }^{18}$ for HDPE liner piping material at $20^{\circ} \mathrm{C}$ the permeabilities can be calculated using the equation

$$
P=D \cdot S
$$

For a polyethylene polymer with low density (low crystallinity), the permeability will be $2.94 \times 10^{-13}$ and for the high density polymer, the permeability will be $4.20 \times 10^{-14}\left(\mathrm{~cm}^{3} \mathrm{~cm} / \mathrm{cm}^{2} \mathrm{~Pa} \cdot \mathrm{s}\right)$.

The leak rate, or flux of $\mathrm{H}_{2}$ gas though the pipe walls during proposed service conditions can then be calculated by evaluating the following equation

$$
J=P \frac{A}{l}\left(p_{0}-p_{1}\right)
$$

where $J$ is the flux, $l$ is the pipe wall thickness, $A$ is the surface area of the pipe exposed to the gas, and $p_{0}$ and $p_{1}$ are the pressures inside and outside the pipe, respectively. The thickness of the liner in the Fiberspar ${ }^{\circledR}$ linepipe is $0.525 \mathrm{~cm}$ and the length of the pipe is assumed to be 1 meter. The area of pipe liner (HDPE) 
exposed to hydrogen gas for this calculation is $3173 \mathrm{~cm}^{2} / \mathrm{m}$. The pressure inside the pipe is assumed to be 103 bar $\left(1.03 \times 10^{7} \mathrm{~Pa}\right)$.

The flux through a polyethylene liner material with high amorphous content (low crystallinity) is calculated as

$$
J=2.94 \times 10^{-13}\left(3173 \mathrm{~cm}^{2}\right) /(0.525 \mathrm{~cm}) *\left(1.03 \times 10^{7}-9.97 \times 10^{4}(\mathrm{~Pa})\right)
$$

where $J=0.018 \mathrm{~cm}^{3} / \mathrm{s}\left(8.09 \times 10^{-7} \mathrm{~mol} / \mathrm{s}\right)$ per meter of pipe. For the high crystallinity PE pipe liner, $J=0.003 \mathrm{~cm}^{3} / \mathrm{s}\left(1.16 \times 10^{-7} \mathrm{~mol} / \mathrm{s}\right)$ per meter of pipe. It is clear from this calculation that as the density of the polymer changes, the crystallinity also changes, which affects the flux of hydrogen through the material. As the crystallinity is decreased from $90 \%$ to $30 \%$, the leakage rate increases six-fold, verifying that crystalline polymer regions provide a barrier to gaseous flux.

\subsection{CONCLUSIONS \& SUGGESTIONS FOR FUTURE WORK}

Several important conclusions can be drawn with respect to transporting hydrogen in fiber-reinforced polymer linepipe:

- Solubility of hydrogen in polyethylene and other similar polymers is very low and it has been shown that the solubility coefficient is relatively independent of polymer types of similar chemistry at a given temperature.

- There are many qualitative structure-property relationships with respect to permeation, which implies that a polymer can be specifically engineered to control permeation. The sample calculations show the drastic changes in hydrogen flux with changes in polymer crystallinity.

- The diffusion coefficient is the main driving force for permeation, and is temperature, pressure, and polymer-gas combination dependent. Diffusion is the physical movement of gas molecules through the polymer free volume.

- There are very few or no gas-polymer interactions during permeation when considering a non-polar gas, such as hydrogen.

- No mechanism exists for hydrogen embrittlement without another catalyst for degradation, such as heat or irradiation.

- There have been several studies on polyethylene, but data for polyphenylene sulfide, which is the other candidate liner material, is severely lacking. Due to their similar chain structure, degree of crystallinity, and chain orientation, it can be concluded that their permeation and solubility behavior in the presence of hydrogen will be similar.

This review shows that there are many opportunities for research and development in the area of hydrogen transport pipe liner materials. One area of interest may be to enhance the barrier properties of polymer liners. This may be accomplished by adding fillers to the current polyethylene polymer that could 
greatly decrease the rate of hydrogen permeation, yet still provide an economically viable option for scale-up. Another area of research may be in specifically engineering a new polymer liner material to enhance barrier properties, such as increasing crystallinity or adding complex side chain structures to inhibit chain movement. Processing methods may also be considered to favorably change polymer chain orientation.

In the absence of a mechanism for degradation of the polymer by exposure to pure hydrogen, it is suggested that permeation and degradation studies be performed on mixed gas streams at pressures similar to that of hydrogen transport service. It would be beneficial to investigate the effects of contaminant gases on the permeation behavior of candidate materials to determine whether selective membrane filtration is necessary in the upstream or downstream operations. It is further suggested that the purity of transported hydrogen be determined so that the proposed pipeline material can be tested in service conditions. It may be that trace contaminants or a carrier gas provide the only mechanism for pipeline degradation and failure.

Finally, it is paramount that future research in this area be focused on the codification of the material and construction of candidate pipeline for use in the proposed hydrogen transport infrastructure. Previous work in the area of utilizing fiber-reinforced pipeline material in the transport of natural gas can be leveraged as a starting point.

\subsection{REFERENCES}

1. Paster, M. Production and Delivery Technology Development Manager, DOE-EE, presentation at the ASME/SRNL Materials and Components for Hydrogen Infrastructure Codes and Standards Workshop and DOE Hydrogen Pipeline Working Group Meeting, Aiken, SC, Sept. 23-26, 2007.

2. Laney, P. Use of Composite Materials in the Transportation of Natural Gas, Document INEEL/EXT-02-00992, Idaho National Laboratory, July 2002.

3. Park, J.; Paul, D. J. Membrane Sci. 125 (1997) 23-39.

4. Klopffer, M.; Flaconneche, B.; Odru, P. Plastics, Rubbers and Composites 36 (2007) 184-189.

5. Michaels, A.; Bixler, H. J. Polymer Science 50 (1961) 413-439.

6. Sperling, L. H. Physical Polymer Science (Third Edition), John Wiley and Sons, Inc.

7. Brandrup, J.; Immergut, Edmund H.; Grulke, Eric A.; Abe, Akihiro; Bloch, Daniel R. Polymer Handbook (4th Edition). John Wiley \& Sons.

8. Tanaka, K.; Kita, H.; Okano, M.; Okamoto, K. Polymer 33 (1992) 585-592.

9. Wang, Z.; Chen, T.; Xu, J. Macromolecules 40 (2007) 3238-3245.

10. Aguado, J.; Serrano, D. P.; Vicente, G.; Sanchez, N. J. Polym. and the Environ. 14 (2006) 375-384.

11. Ubrich, E.; Fafet, A.; Robert, E.; Serpe, G. Revue del Institut Francais du Petrole 49 (1994) 165-175.

12. Fayolle, B.; Colin, X.; Audouin, L.; Verdu, J. Polymer Degradation and Stability 92 (2007) 231-238.

13. Wypych, G. (1995). Handbook of Material Weathering (Second Edition). ChemTec Publishing. 
14. Painter, Coleman (1997) Fundamentals of Polymer Science (Second Edition), CRC Press, LLC.

15. Kamiya, Y. et al. Macromolecules 33 (2000) 3111-3119.

16. Kamiya, et al. J. polym. sci., Part B, Polym. Phys. 35 (1997) 1049-1053.

17. Molyneux, P. J. Applied Polymer Science 79 (2001) 981-1024

18. Humpenöder, J. Cryogenics 38 (1998) 143-147. 


\subsection{APPENDIX- BASIC MODEL FOR HYDROGEN TRANSPORT IN POLYMERS}

Regardless of the permeating species (liquid or gas), the process of permeation through a polymeric material takes place in three distinct steps. The permeant must first absorb or condense onto the polymer surface, then diffuse through the polymer via chemical potential gradients or pressure differences, and finally desorb or evaporate from the polymer into the atmosphere at the opposite side of the film. Unlike permeation of a gas through a metal, molecular gases does not dissociate into atomic species before absorption in a polymer.

Diffusion does not take place along a route of lattice site jumps, but rather through the ever-changing free volume movement of the polymer long chain molecules. There is no common model for the dynamics of polymer chain movement; however, there is general agreement that the chains are sufficiently perturbed during diffusion to allow a penetrant molecule to reside between chains. The calculation of the associated energies needed for a permeant molecule to jump between sites is a topic of current research ${ }^{17}$. This generally accepted model of diffusion is termed "solution-diffusion" and can be described mathematically as follows.

The solution of gas molecules in polymers is described by:

$$
S=S_{0} \cdot p \cdot e^{\frac{-\Delta H}{R T}}
$$

in which $p$ is the partial pressure of the gaseous species, $\Delta H$ the heat of solution, $R$ the gas constant, $T$ the absolute temperature, and $S_{0}$ the solubility constant. The heat of solution, $\Delta H$, varies with the dissolving molecule, polymer and temperature. Diffusion of a permeating species is described by Fick's Law (one dimensional case):

$$
J=-D \frac{\partial c}{\partial x}
$$

and

$$
\frac{\partial c}{\partial t}=D \frac{\partial^{2} c}{\partial x^{2}}
$$

in which $J$ is the flux (amount of matter diffusing per unit area per unit time), $D$ the diffusion coefficient, $c(x, t)$ is the concentration of the diffusing molecule (function of time, $t$, and position in the polymer, $x$ ). The relation, $\frac{\partial c}{\partial x}$, is the concentration gradient, the driving force for diffusion. This assumes the solution follows Henry's law, which is usually true for pure permeating species. If the solubility is not linear with partial pressure, the driving force for diffusion is the 
gradient of the chemical potential of the molecule in the polymer, $\frac{\partial \mu}{\partial x}$. The diffusion coefficient normally varies exponentially with temperature:

$$
D=D_{0} \cdot e^{\frac{-Q}{R T}}
$$

in which $Q$ is termed the activation energy of diffusion and $D_{o}$ is the preexponential factor.

In principle, Eq. (3) can be solved, with initial and boundary conditions, to find the concentration as a function of time and position in the film, slab, etc. The exit flux is calculated using Eq. (2). Analytical solutions to Eq. (3) exist for only a few specific cases. Finite difference methods can be used to numerically solve arbitrary boundary and initial conditions. However, for the case of gas contained in a polymer-lined pipe at constant pressure and temperature, which is of interest to expected conditions for transport of hydrogen in underground pipelines, a simplified case can be applied. The steady state approximation, which would be quite accurate in this case, becomes valid after a characteristic time. Using this approximation, the concentration no longer changes with time, implying that the concentration varies linearly with position in the liner thickness:

$$
c(x)=S\left(1-\frac{x}{L}\right)
$$

in which the concentration varies from the solubility limit at the inner surface, $S$, to zero at the outer surface. $L$ represents the thickness of the film, slab, liner, etc. From Fick's First Law the steady state flux is:

$$
J=-D\left(\frac{S}{L}\right) .
$$

Equation (6) represents the flux of molecular gas through the polymer liner into the environment, which is the amount of gas permeating per unit area per unit time. The permeability is defined as:

$$
\Phi=D \cdot S
$$

and so the steady state flux is:

$$
J=\frac{-\Phi}{L}
$$

The approximate time to achieve steady state permeation is given by ${ }^{5}$ : 


$$
\tau \cong 0.45 \frac{L^{2}}{D}
$$

From Eq. (6), the factors minimizing the flux, $J$, of permeating gas are minimizing diffusivity and solubility and maximizing the liner thickness. The thickness is ultimately determined by factors such as cost and ease of processing. Minimizing $D$ and $S$ entails material selection for the given molecule permeating the barrier. 